\title{
Contact Lens Following Corneal Perforation Injury-A Case Report
}

\author{
Patrick Joi Tsang Shum* \\ New Taipei Eye Clinic, Taiwan \\ *Corresponding author: Patrick Joi Tsang Shum, New Taipei Eye Clinic, New Taipei City 221,Taiwan
}

\begin{tabular}{|c|c|}
\hline ARTICLE INFO & ABSTRACT \\
\hline Received: 幽 January 26, 2019 & A 72-year-old male was a victim in an accident and rupture of eyeball with traumatic \\
\hline Published: 㫵February 06, 2019 & $\begin{array}{l}\text { cataract was noted on the left eye. Primary repair of corneal perforation and lensectomy } \\
\text { were performed but no intraocular lens was inserted concomitantly in the emergent }\end{array}$ \\
\hline $\begin{array}{l}\text { Citation: Patrick Joi Tsang Shum. Con- } \\
\text { tact Lens Following Corneal Perfora- } \\
\text { tion Injury-A Case Report. Biomed J } \\
\text { Sci \& Tech Res 14(1)-2019. BJSTR. } \\
\text { MS.ID.002503. }\end{array}$ & $\begin{array}{l}\text { management, which left a corneal scar on the aphakic eye eventually. For the improvement } \\
\text { of vision in this unilateral traumatic aphakes, an intralimbal RGP contact lens made with } \\
\text { high DK material was prescribed for vaulting over the cornea entirely to provide a good } \\
\text { cornea-to-lens relationship and prevent the aniseikonia. The patient felt satisfied because } \\
\text { of good centration, optimal apical clearance, comfort and acceptable corrected visual } \\
\text { acuity. }\end{array}$ \\
\hline
\end{tabular}

Keywords: Intralimbal RGP Contact Lens; Aphakia; Irregular Astigmatism

Abbreviations: RGP: Rigid Gas Permeable ; UCVA: Uncorrected Visual Acuity ; BCVA: Best Corrected Visual Acuity ; IOL: Implantation of Intraocular Lenses

\section{Introduction}

Corneal perforation injuries can often cause corneal scars and aphakia because of corneal sutures and lensectomy during the emergent surgical repair which result in significant visual impairment, mainly due to the loss of the crystalline lens and irregular corneal astigmatism. The presence of an irregular astigmatism prevents optimal improvement in visual acuity with spectacle correction. Martin and de Juan [1] reported that visual improvement with contact lenses in unilateral aphakia was significantly greater than with spectacles. Rigid gas permeable (RGP) contact lenses improve visual acuity by providing a smooth refracting surface negating the irregular astigmatism due to scar $[2,3]$.The tears flow underneath the RGP lens provide a tear layer between the contact lens and the cornea which reduce the scattering effect induced by the irregular surface of the cornea [4]. For unilateral aphakia, an added advantage could be the lower degree of unilateral magnification induced by contact lenses in comparison with spectacles. Therefore, RGP contact lenses are an ideal nonsurgical alternative to provide increased visual acuity in patients with irregular corneal astigmatism [5].

\section{Case Presentation}

A 72-year-old male was a victim in an accident 3 years ago and rupture of eyeball with traumatic cataract was noted on the left eye at that time. He was admitted at a medical center where the primary repair of corneal perforation wound and lensectomy were performed but no intraocular lens was implanted concomitantly in this emergent management. This patient came to our clinic and asked for further evaluation 3 years later. A detailed work-up was carried out at our clinic, which included uncorrected visual acuity (UCVA), best corrected visual acuity (BCVA), autorefractometry, non-contact tonometry, corneal topography, slit lamp bio microscopy and indirect ophthalmoscopy. The uncorrected visual acuity was $20 / 100$ and 20/2000 on the right and left eyes respectively. With a subjective refraction of $-1.00-2.5 \times 950 D$ and $+12.00-0.75 \times 1000$ S, the best-corrected visual acuity was 20/50 on the right and 20/100 on the left side respectively. For the left eye, slit lamp bio microscopy showed a whitish scar about $1 \mathrm{~mm}$ away from the center of cornea nasally (Figure 1). The anterior chamber depth was not shallow, but the pupil was irregular shaped 
and mid-dilated. There was a posterior synechia between the iris and the residual lens capsule around the nasal part of the pupil, but no implantation of intraocular lens was noted (Figure 2). Dilated fundus examination was unremarkable for each eye which ruled out the existence of active fundus pathology, such as retinal detachment, retinal hemorrhage, cystoid macular edema, macular hole and other active retinopathies.

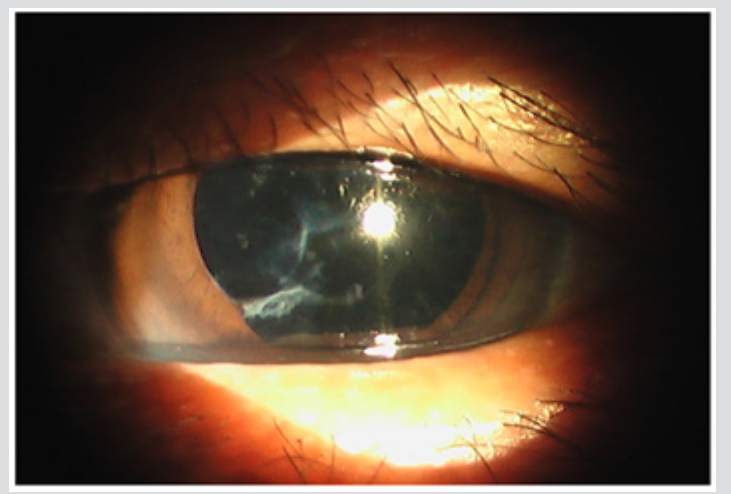

Figure 1: There was a whitish scar on cornea of the left eye being $1 \mathrm{~mm}$ away from the center nasally.

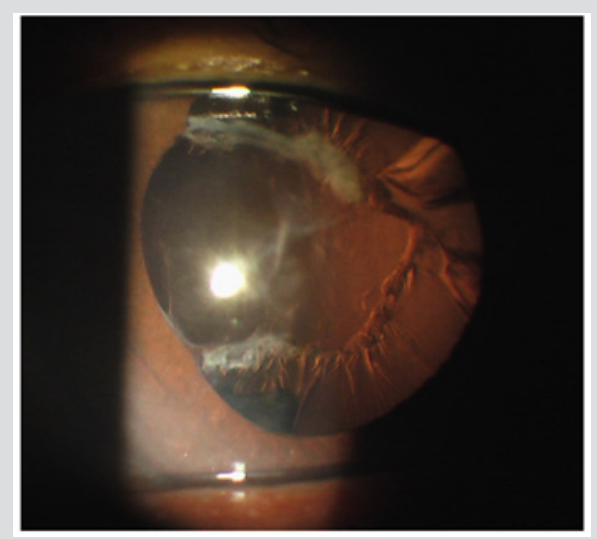

Figure 2: There was a posterior synechiae between the iris and the residual lens capsule around the nasal part of the pupil, but no implantation of intraocular lens was noted.

The corneal topography of the traumatized eye showed topographical steepening over the corneal scar but not much astigmatism was shown on the simulated keratometry readings (Figure 3). However, the best-corrected visual acuity was not good because of significant irregular astigmatism at the center of the left eye. Contact lens fitting was performed according to refraction and keratometric values. Rigid gas permeable contact lens of high DK value (Boston XO) was fitted on the left eye by trial and error method and the corneal topographic $\mathrm{K}$ value at $3 \mathrm{~mm}$ zone was taken into consideration. To start with, the mean keratometric (K) value was first evaluated as the base curve of the contact lens. The fit was adjusted based on the cornea-to-lens relationship by analyzing the fluorescein pattern in the tear layer. A fluorescein pattern that showed clearance from the center to the peripheral region with coverage on the scar surface was considered to be ideal.

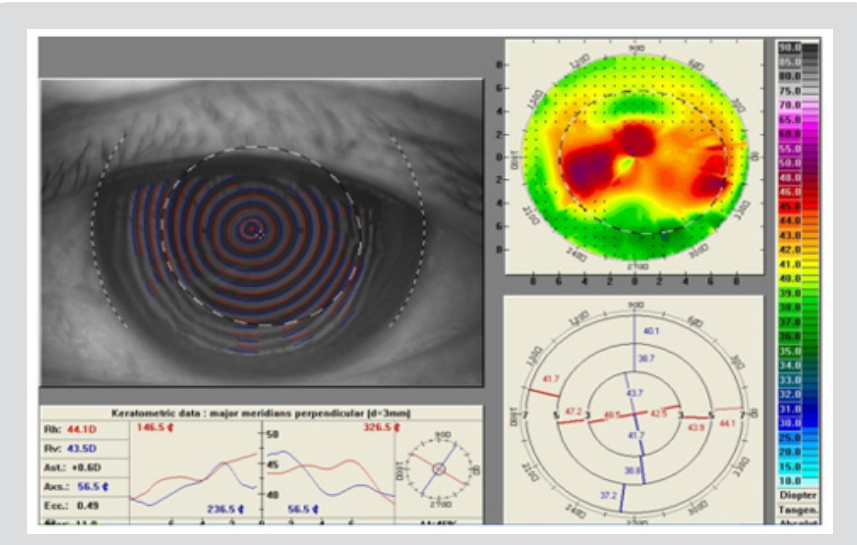

Figure 3: The corneal topography of the traumatized eye showed topographical steepening over the corneal scar.

The lens was fit slightly steeper than usual and a contact lens with large overall diameter of $12 \mathrm{~mm}$ was selected to achieve superior alignment fit. The fit was evaluated on parameters of centration, movement, coverage and the fluorescein pattern to achieve the best fit. Over refraction was performed to get the final power of contact lens and the best contact lens corrected visual acuity was recorded for each new fit. The final lens had a 45.2D base curve, $0.1 \mathrm{~mm}$ central thickness, $+10.5 \mathrm{D}, 12 \mathrm{~mm}$ diameter and the corrected visual acuity with RGP contact lens was $20 / 40$ on the left eye (Figure 4).

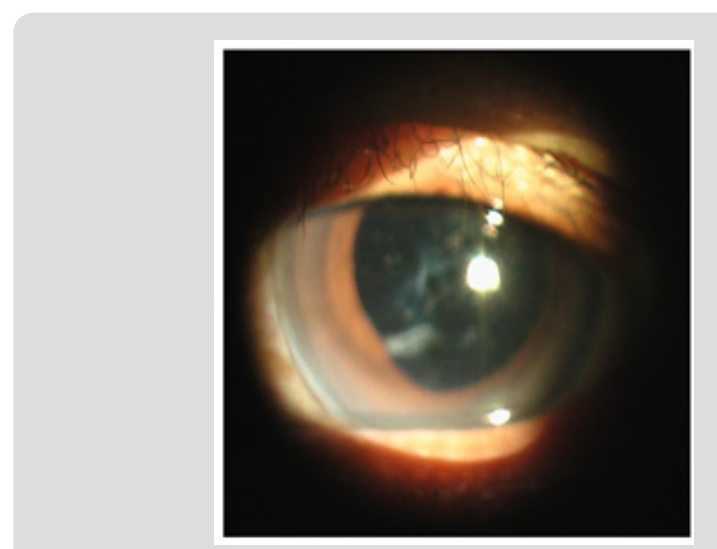

Figure 4: The traumatized eye was fitted with an intralimbal RGP contact lens.

\section{Discussion}

Corneal scars following perforating corneal injuries cause significant visual reduction, which is mainly due to the obstruction of light rays by the opacity and also because of the irregular corneal astigmatism that results from the scar [6]. The severity of vision impairment may be determined by several factors including the size, density and location of scar. Corneal sutures and subsequent scarring from previous wounds frequently produce high astigmatism $[7,8]$ which reduce the visual acuity especially when the scars encroach upon the visual axis [9]. According to a previous study, larger corneal wounds in close proximity to the visual axis produce the greatest amount of astigmatism and influence 
the visual acuity profoundly [4]. If a traumatic corneal wound is within the visual axis due to a full-thickness perforation wound, and causing a severe visual reduction, penetrating keratoplasty is often needed to excise the corneal scar and remove the irregular astigmatism associated with the scar [10]. Aphakia is a medical condition characterized by an absence of the crystalline lens of the eye. It is an anomaly of the binocular visual process that affects the patient's perceptual judgment. When a person having one aphakic and one complete eye wears conventional corrective spectacles, the symptoms including asthenopia, headache, diplopia, vertigo, photophobia, and other distressing may develop. The most common cause is the magnification from aphakic spectacles in correction of anisometropia. This difference in magnification produces different sized retinal images and creates image disparity which results in a fusional inability for the two eyes to work together.

The actual magnification is determined by the exact power of the aphakic spectacles. There is approximately $2 \%$ of magnification for each diopter of power in the spectacles. As the average aphakic spectacle is about $12.5 \mathrm{D}$, the image that is formed on the patient's retina to be roughly 25\% larger than the image formed with the crystalline lens [11]. Furthermore, the magnification from aphakic spectacles can cause other optical aberrations, such as a ring scotoma, jack-in-the-box phenomenon, and a pincushion distortion [12]. The vision through aphakic spectacle is distorted in this way, which makes rectangular objects, such as doors and boxes, appear like a pincushion. Therefore, the distance judgment is impaired, and it may cause clumsiness in performance of simple tasks. In aphakes with traumatic history, the visual impairment is mainly due to the loss of the crystalline lens and irregular corneal astigmatism. Usually, the visual improvement with spectacles and soft contact lens is not as good as phakic and pseudophakic eyes. This is perhaps because of the irregular astigmatism and corneal scar developed on these eyes. However, the improvement with RGP contact lenses in these eyes was significantly greater over spectacle correction [9]. For aphakes, the added advantage could be the less amount of unilateral magnification induced by contact lens in comparison to spectacles [6].

The aim of treating unilateral aphakia with inadequate capsular support after trauma is to increase visual acuity and improve quality of life. Several modalities are available for the correction of visual impairment in traumatic aphakia including secondary implantation of intraocular lenses (IOL), spectacles, scleral lenses, soft contact lenses and RGP contact lenses. Obviously, spectacles were not suitable for cases with unilateral aphakia due to severe anisometropia (aniseikonia) [13]. Secondary implantation of IOL is an insertion of a lens in an eye without crystalline lens inside the eyeball. In the absence of capsular support, surgical options to correct aphakia include implantation of an angle-supported anterior chamber IOL, anterior chamber iris-fixated IOL, retro pupillary irisfixated IOL, iris-sutured posterior chamber IOL, and scleral-fixated posterior chamber IOL. [14-16] All techniques have advantages and disadvantages, and no consensus exists on the indications for and relative efficacy and safety of these options [17]. However, secondary IOL implantations can cause many complications, including corneal edema, hyphema, glaucoma, ciliary choroidal body hemorrhage, retinal detachment, retinal tears, vitreous hemorrhage, cystoid macular edema, lens tilting, decentration, vitreous prolapse into the anterior chamber, endophthalmitis and damage to the iris [13]. Azhar also conducted a study regarding the complications during secondary IOL implantation and he concluded that hemorrhage in $13.9 \%$ and vitreous loss occurred in $3.9 \%$ patients.

Cystoid macular edema occurred in 5.8\% and corneal decompensation in $19.2 \%$ of patients [18]. Many techniques have been described for correcting aphakia with inadequate capsular support, but it is still a challenge for ophthalmic surgeons. In this case, the visual impairment may not be corrected by the secondary implantation of IOL as a high irregular astigmatism induced by the scar which is located at the center of the cornea. Previous studies confirm that the visual acuity of patients wearing contact lenses is significantly better than that of those with spectacles, [19] especially in the treatment of unilateral aphakia [20,21]. The contact lenses provide a more regular refractive surface and reduction in vertex distance which eliminate the visual distortion and create a clear retinal image [22]. Therefore, contact lenses can be served as an important therapeutic role in visual rehabilitation [23]. When considering a contact lens to correct the astigmatism, the type of contact lens must be chosen. Soft contact lenses are made of a flexible plastic material, which is normally hydrophilic and generally more comfortable than rigid contact lenses. However, when a soft lens is placed on the eye, the lens conforms to the anterior corneal shape, and the refractive effect of the tears between the contact lens and the cornea is minimized [24] that soft contact lens is inadequate to correct the irregular astigmatism.

Rigid gas permeable contact lens is available for treating refractive abnormalities in traumatic unilateral aphakia. It masks significant amount of irregular astigmatism because the tear film underneath the contact lens can neutralize the surface irregularity [6] which provides a smooth refractive surface [2] and permits an improvement of the visual acuity [24]. Furthermore, for the corneal scar, RGP contact lenses provide significant improvement in visual acuity in eyes with nebular and nebulo-macular corneal opacity [25]. When the corneal scar is closer to the visual axis, the improvement from spectacle-corrected visual acuity to RGP contact lens visual acuity is more profound [9]. This procedure is effective, safe and tolerable. Therefore, the RGP contact lenses were suggested to fit this patient in order to have a good visual correction. There are some guidelines in approach to managing the irregular cornea with contact lenses. First, oxygen delivery to the compromised cornea should be maximized by designing the lenses in the most permeable material that allows for visual stability. Second, the 
lenses should vault the diseased or damage tissue by aiming for an apical or overall corneal clearance fluorescein pattern. Third, good centration, movement, and tear exchange should be sought to preserve corneal integrity and optimize visual acuity. Finally, toric base curves should be avoided in the correction of irregular astigmatism [26,27].

The traditional contact lens fitting procedure for irregular shaped corneas is more challenging than for regular shaped ones $[28,29]$. as the trial lenses may not match the shape of irregular cornea properly which causes the ocular discomfort, poor centration and visual instability. Ocular discomfort was the main reason for dropout of contact lens treatment [30].The high DK RGP lens materials have been proved to fit with much larger-diameter designs without concerning the hypoxic complications. Therefore, scleral lenses are designed to vault over the entire corneal surface and rest on the white of the eye which provide more comfort and better centration, but they still cause a palpable degree of sensation, appear bulky and some people are intimidated by their large size. The scleral lenses cover a large area of the anterior globe and considerably reduce the transmission of oxygen to the cornea, although the high DK material has been applied [13]. Smaller lenses are only indicated for small central or paracentral scars as the overall diameter (OAD) is ranged from 7.9 to $10 \mathrm{~mm}$ in interpalpebral lenses. For larger scars secondary to trauma or laceration, which create more global irregular astigmatism, an intralimbal design is often necessary that the size (OAD) is 10 to $12.5 \mathrm{~mm}$ in diameter as it provides the optimal lens centration, minimal glare, less corneal bearing forces, and rare lens displacement [31]. It tends to center better than smaller lenses as a result of greater pressure distribution over the midperipheral and peripheral cornea. These lenses are more comfortable than the smaller designs due to both the good centration and less movement with the blink than the small-diameter designs [32].

Generally, intralimbal lenses have a large optical zone that it may be helpful on the visual correction when the lens decenters for a little bit. This is especially important for corneas with large or displaced areas of irregularity. Because of their size, the central optics are better maintained over the pupil without displacement, in addition, these lenses may offer improved comfort and vision for patients [33]. In this case, the corneal scar was closed to the visual axis of the left aphakic eye that we prescribed an RGP contact lens with using the high DK material (Boston XO) which was indicated for the diseased or scarred cornea. An intralimbal design was decided to vault over the cornea entirely to prevent mechanical interaction between the scar and posterior lens surface. The patient felt satisfied because of good centration, optimal apical clearance, comfort and acceptable corrected visual acuity. It is a challenge for the ophthalmologist to correct the irregular astigmatism. In most cases, spectacles with adequate correction are not enough to improve the visual acuity, leaving contact lenses as the sole option for treatment instead of surgery. In conclusion, the RGP contact lenses do play an important role in the treatment of post-traumatic irregular astigmatism because they improve the visual acuity and exhibit lower risks than surgical procedures.

\section{References}

1. Martin R, de Juan V (2007) Reverse geometry contact lens fitting in corneal scar caused by perforating corneal injuries. Cont Lens Anterior Eye 30(1): 67-70.

2. Dada VK, Agarwal LP, Martin S, Harris RL (1975) Visual Acuity improvement in eyes with corneal scars fitted with contact lenses. Am J of Optom Physiol Optics 52(3): 211-215.

3. Elliot DB, Whitaker D (1991) Factors affecting light scattering in contact lens wearers. Optom and Vis Sci 68(8): 629-633.

4. Jupiter DJ, Katz HR (2000) Management of irregular astigmatism with rigid gas permeable contact lenses. CLAO J 26(1): 14-17.

5. Titiyal JS, Sinha R, Sharma N, Sreenivas V, Vajpayee RB (2006) Contact lens rehabilitation following repaired corneal perforations. BMC Ophthalmol 6: 11-14.

6. Caroline P, Andre MP (2010) Contact Lenses Following Corneal Trauma Contact Lens Spectrum 25: 56.

7. Navon SE (1997) Topography after repair of full-thickness corneal laceration. J Cataract Refract Surg 23(4): 495-501.

8. Smith SD, Navon SE (1994) Computerized topography of a full-thickness corneal laceration. Ophthalmic Surg 25(9): 630-632.

9. Zheng B, Shen L, Walker M K, Zhang Z, Zheng J, et al. (2015) Clinical Evaluation of Rigid Gas Permeable Contact Lenses and Visual Outcome After Repaired Corneal Laceration. Eye \& Contact Lens 41(1): 34-39.

10. Sonsino J, Ewald MD (2010) Corrective Options for Irregular Cornea. Contact Lens Spectrum 25: 26-35.

11. Yanoff M, Duker JS (2009) Ophthalmology. In: Yanoff M, Duker JS(Eds.), ( $3^{\text {rd }}$ edn.), Elsevier Health Sciences, USA, pp. 413-414.

12. Nema HV, Nema N (2011) Textbook of Ophthalmology. In: Nema HV, Nema N (Eds.), (6 ${ }^{\text {th }}$ edn.), Jaypee-Highlights Medical Publisher Inc, Panama, pp.287.

13. Wei Ling Luo, Jian Ping Tong, Ye Shen (2012) Rigid gas-permeable contact lens for visual rehabilitation in aphakia following trauma. Clin Exp Optom 95(5): 499-505.

14. Zeh WG, Price FW (2000) Iris fixation of posterior chamber intraocular lenses. J Cataract Refract Surg 26(7): 1028-1034.

15. De Silva SR, Arun K, Anandan M, Glover N, Patel CK, et al. (2011) Iris-claw intraocular lenses to correct aphakia in the absence of capsule support. J Cataract Refract Surg 37(9): 1667-1672.

16. Dick HB, Augustin AJ (2001) Lens implant selection with absence of capsular support. Curr Opin Ophthalmol 12(1): 47-57.

17. Evereklioglu C, Er H, Bekir NA, Borazan M, Zorlu F (2003) Comparison of secondary implantation of flexible open loop anterior chamber and scleral-fixated posterior chamber intraocular lenses. J Cataract Refract Surg 29(2): 301-308.

18. Azhar RF (1978) Secondary implantation of intraocular lenses. Ann. Ophthalmology 10: 658-662.

19. Kanpolat A, Ciftci OU (1995) The use of rigid gas permeable contact lenses in scarred corneas. CLAO J 21(1): 64-66.

20. Boyd HH (1979) Hard contact lens corrections in aphakia. Ophthalmology 86(3): 399-402.

21. Jain IS, Mohan K, Gupta A (1985) Unilateral traumatic aphakia in children: role of corneal contact lenses. J Pediatr Ophthalmol Strabismus 22(4): 137-139. 
22. Guyton DL (1977) Prescribing cylinders: the problem of distortion. Surv Ophthalmol 22(3): 177-188.

23. Resnick SA (2008) Contact Lens Management of the Irregular Cornea. Contemporary Optometry 11(6): 1-6.

24. Herranz MR, Zarzuelo GR , Herraez V de Juan (2012) AstigmatismOptics, Physiology and Management pp. 157-180.

25. Titiyal JS, Das A, Dada VK, Tandon R, Ray M, et al. (2001) Visual performance of rigid gas permeable contact lenses in patients with corneal opacity. CLAO J 27(3): 163-165.

26. Zadnik K, Barr JT (1999) Diagnosis, Contact Lens Prescribing and care of the Keratoconus Patient. Butterworth Heinemann, USA, pp. 27-42.

27. Anderson B, Edmonds S, Bennett E (2007) Case Grand Rounds: GP Keratoconus Patients. Contact Lens 22: 1-4.

ISSN: 2574-1241

DOI: 10.26717.BJSTR.2019.14.002503

Patrick Joi Tsang Shum.Biomed J Sci \& Tech Res

(C) This work is licensed under Creative Commons Attribution 4.0 License

Submission Link: https://biomedres.us/submit-manuscript.php
28. Yeung KK, Olson MD, Weissmann BA (2002) Complexity of contact lens fitting after refractive surgery. Am J Ophthalmol 133(5): 607-612.

29. Zhon AJ, Kitomurak K, Weissmann BA (2003) Contact lens care in keratoconus. Cont Lens Anterior Eye 26(4): 171-174.

30. Fujita H, Sano K, Sasaki S, Ohno Matsui K, Tanaka T, et al. (2004) Ocular discomfort at the initial wearing of rigid gas permeable contact lenses. Jpn J Ophthalmol 48(4): 376-379.

31. Resnick SA (2008) Contact Lens Management of the Irregular Cornea. Contemporary Optometry 11(6): 1-6.

32. DeNaeyer GW (2011) Managing compromise with large-diameter corneal GPs. Contact Lens Spectrum (1): 20.

33. Sclafani LA (2007) Fitting Contact Lenses to Irregular Corneas. Contact Lens Spectrum, 34-39.

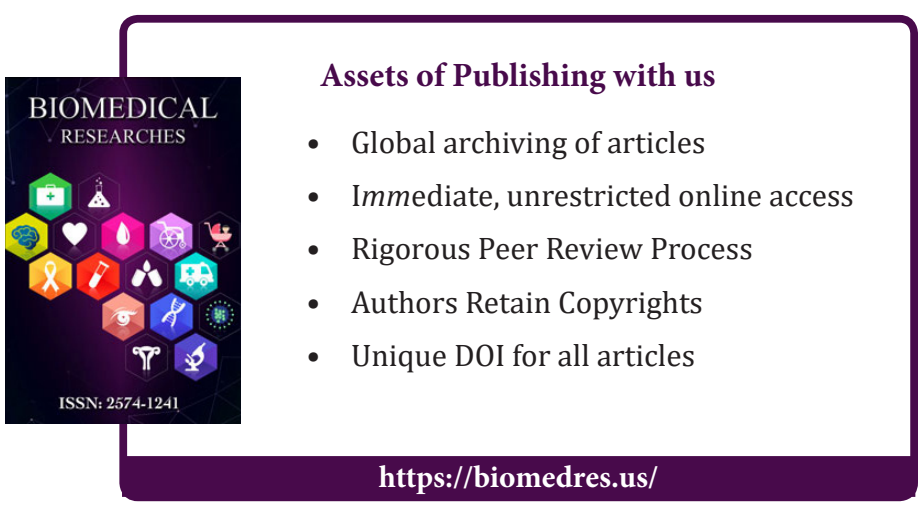

